\title{
Metamorphic antimonides on GaAs for thermophotovoltaic devices
}

\author{
A. M. Sanchez, ${ }^{1}$ J. M. Ripalda, ${ }^{2 *}$ A. G. Taboada, ${ }^{2}$ A. Rivera, ${ }^{2}$ B. Alén, ${ }^{2}$ D. Fuster, ${ }^{2}$ \\ Y. González, ${ }^{2}$ L. González, ${ }^{2}$ G. Balakrishnan ${ }^{3}$
}

\author{
1 Physics Department, University of Warwick, Coventry CV4 7AL, United Kingdom \\ 2 Instituto de Microelectrónica de Madrid, CNM, CSIC, Isaac Newton 8, Tres cantos 28760, Spain \\ 3 Center for High Technology Materials, University of New Mexico, Albuquerque, New Mexico 87106, USA
}

\begin{abstract}
1. Abstract
The growth of metamorphic III-V semiconductors on substrates of dissimilar lattice parameter is of interest for the engineering of optoelectronic devices for which the cost of the substrate would otherwise limit the applicability of the material. Such is the case of the antimonides, which are very appropriate for small band gap photovoltaics or thermophotovoltaics. To further increase the efficiency of tandem solar cells (typically based on Ge or GaAs substrates) it is necessary to integrate materials with band gap $<1.4 \mathrm{eV}$. Metamorphic InGaAs has been successfully integrated in GaAs based tandems, but requires the growth of thick step graded buffers of InGaP. ${ }^{1}$ Here we present a study on the nucleation and subsequent growth of AlSb on GaAs (001), and compare our results with the more extensively studied case of $\mathrm{GaSb}$ on $\mathrm{GaAs}^{2,3}$ and the case of AlSb grown on $\mathrm{Si}^{4}$ We have observed the nucleation of almost completely relaxed AlSb islands after $0.11 \mathrm{ML}$ of AlSb. The lattice parameter of the metamorphic material is slightly smaller than that of bulk AlSb. Measuring the deformation of the substrate during growth, we have observed that residual stress accumulates due to the incomplete plastic relaxation at the interface. Transmission Electron Microscopy (TEM) images show almost all of the plastic relaxation accommodated by an array of $90^{\circ}$ misfit dislocations at the interface (IMF). A very small fraction of the plastic relaxation is accommodated by stacking faults (in the case of AlSb) or threading dislocations (in the case of $\mathrm{GaSb}$ ). We have observed InAs layers grown on $\mathrm{GaSb}$ to be quite effective filters of threading dislocations, but stacking faults in AlSb propagate undisturbed though thin InAs and $\mathrm{GaSb}$ layers.
\end{abstract}

\section{Experimental details}

After oxide desorption and buffer layer growth on
$\mathrm{GaAs}(001)$, the sample is exposed to $\mathrm{Sb}_{2}$ at $600^{\circ} \mathrm{C}$ until a $\mathrm{x} 8$ periodicity is observed in the grazing electron diffraction (RHEED) pattern along the (1-10) azimuth, this being indicative of an Sb terminated GaAs surface. The substrate temperature is then lowered to $500^{\circ} \mathrm{C}$ and antimonide growth is started at $0.25 \mathrm{ML} / \mathrm{s}$ in $\mathrm{Sb}$ rich conditions. The substrate dimensions are typically $20 \times 4 \times 0.1 \mathrm{~mm}$ to maximize the deflection of the susbtrate due to the accumulated stress. The deflection is measured by comparing the angle of reflection a two laser beams, one of them incident on one end that is firmly held to the substrate holder, and the other incident on the free standing end of the substrate. TEM characterization has been carried out on a Jeol 2000FX microscope operating at $200 \mathrm{kV}$. After cleavage along $<110>$ directions, samples were prepared for cross sectional and planar TEM examination by mechanical and ion beam thinning.

\section{Results}

The in situ accumulated stress measurements indicate that about $10 \%$ of the lattice mismatch is accommodated elastically. The elastic/plastic relaxation fraction is much higher during the first ML of antimonide growth on GaAs. This is in contrast to the RHEED measurements, which seem to indicate a nearly instantaneous and complete relaxation of the lattice parameter. This apparent discrepancy is due to the fact that the RHEED measurement is predominantly probing the plastically relaxed islands sticking out of the surface, whereas the stress measurements are more representative of the whole surface. This is consistent with the observation by Huang et al. of coexisting regions with and without the IMF array during the initial stages of antimonide growth. ${ }^{3}$ The TEM shows clear evidence of the IMF both in planar view and cross section, thus the results presented here are probably relevant to other cases of IMF metamorphic growth.

\footnotetext{
* Presenting author.
} 


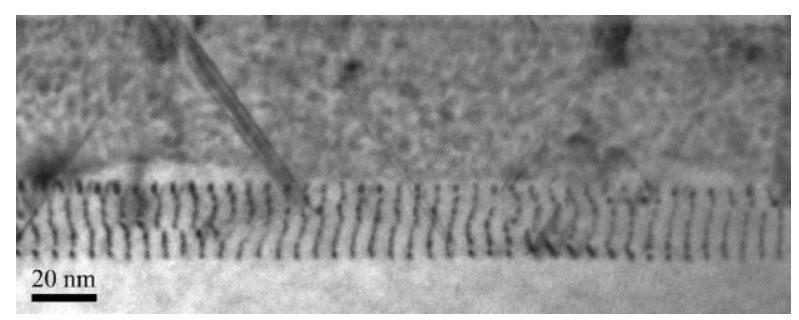

Fig. 1. Bright filed 220 TEM image of the misfit dislocation array at the AlSb/GaAs interface.

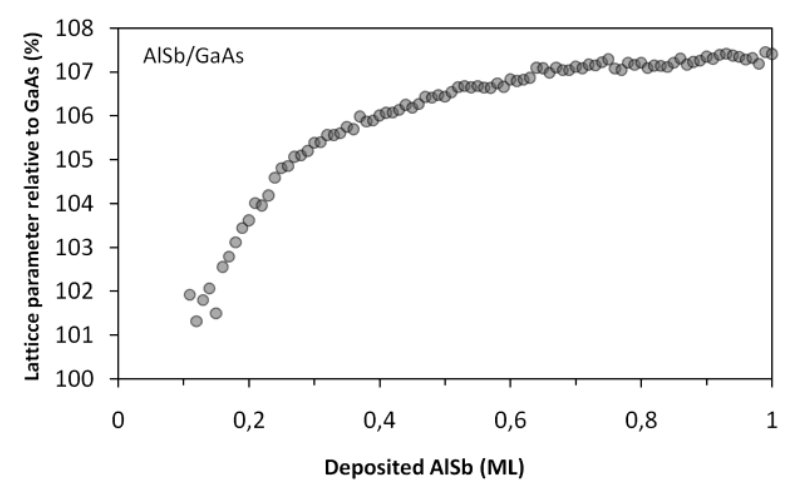

Fig. 2. Evolution of the in plane lattice parameter as a function of the deposited AlSb thickness as measured from the spacing of the RHEED pattern along the [1-10] direction.

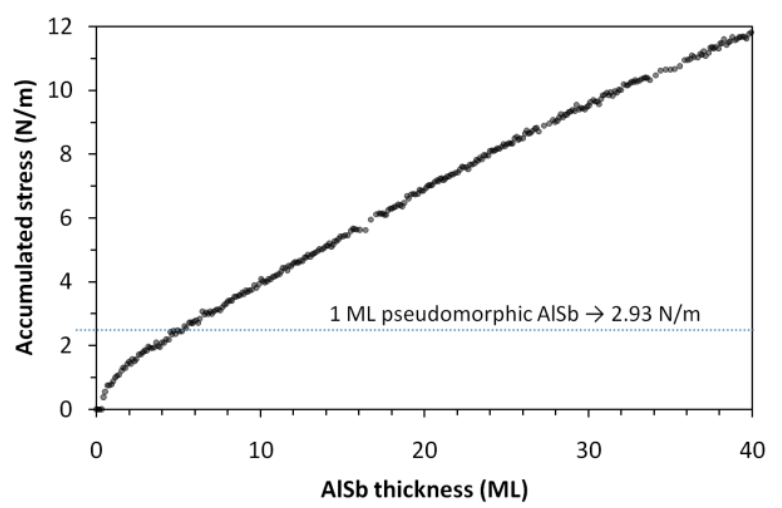

Fig. 3. Acccumulated stress as measured from the substrate deflection during growth of AlSb on GaAs.

\section{References}

1 J. F. Geisz, et al., Appl. Phys. Lett. 91, 023502 (2007).

2 S. H. Huang, et al., Appl. Phys. Lett. 88, 131911 (2006).

3 S. Huang, G. Balakrishnan, D. Huffaker, J. Appl. Phys. 105, 103104 (2009).

${ }^{4}$ G. Balakrishnan et al. Appl. Phys. Lett. 86, 034105 (2005). 